\title{
Assessment of Immuno-Histochemical Expression of MBD1 in Colorectal Adenocarcinoma and Its Correlations with Prognostic Factors
}

\author{
Sholeh Ghaedamini ${ }^{1}$, Mehdi Nikbakht ${ }^{1}$, Mitra Soleimani ${ }^{1, *}$ \\ 1. Department of Anatomical Sciences, \\ Isfahan University of Medical Sciences, \\ Isfahan, Iran

\section{* Corresponding Author:} \\ Mitra Soleimani, Ph.D \\ Hezar jarib, Isfahan University of Medical \\ Sciences, Isfahan, Iran \\ Telefax: + 983137929026 \\ Email: mitsolni@gmail.com \\ Received: 20 Jul. 2019 \\ Accepted: 09 Dec. 2019

\section{ABSTRACT}

\section{BACKGROUND} \\ MBD1, the largest member of methyl binding domain family, has the most downregulated \\ mRNA expression and upregulated methylation levels in advanced colorectal cancer (CRC). In this \\ study, we evaluated the immune-histochemical expression of MBD1 in CRC and assessed its correlation \\ with clinicopathological features to study its prognostic value in CRC.

\section{METHODS} \\ A total of 60 samples of $\mathrm{CRC}$, from patients who underwent surgical gastroenterology operations, \\ were randomly selected. The samples included one tumor-rich section per case and one adjacent \\ tumor-free section as a normal control for that case. Then, immunohistochemistry (ICH) was performed \\ for MBD1 protein on all samples and the expression of MBD1 was analyzed in cancerous and \\ normal samples. In the next step, the correlation between MBD1 and clinicopathological features \\ including age, sex, location of the tumor, grade, and stage were evaluated.

\section{RESULTS} \\ The expression of MBD1 protein had a significant downregulation in cancerous samples compared \\ with normal control samples. This downregulation increased corresponding to both grade and stage \\ of cancer. However, no correlation was seen between the expression of MBD1 and sex, age and \\ location of the tumor.

\section{CONCLUSION} \\ MBD1 protein may be considered as a protein marker in the detection of $\mathrm{CRC}$ and its progression. \\ KEYWORDS: \\ CRC, MBD1, Clinicopathological features, ICH

\section{Please cite this paper as:} \\ Ghaedamini S, Soleimani M, Nikbakht M. Assessment of Immuno-Histochemical \\ Expression of MBD1 in Colorectal Adenocarcinoma and Its Correlations with Prognostic \\ Factors. Middle East J Dig Dis 2020;12:39-44. doi: 10.15171/mejdd.2020.162.

\section{INTRODUCTION} \\ Colorectal cancer (CRC) is the third leading cause of death among all cancers in \\ men and women. ${ }^{1}$ Approximately 800,000 new CRC cases are detected worldwide \\ every year. ${ }^{2}$ The etiopathogenesis of CRC is a complex and multistep process, \\ which is characterized by histopathological precursor lesions and molecular genetic \\ alterations. ${ }^{3}$ CRC is caused by several genetic and epigenetic alterations. ${ }^{4}$ Tumor \\ suppressor genes along with oncogenes have a crucial role in the occurrence and \\ development of cancer. ${ }^{5}$ In general, normal control of cell division is impaired
}


Table 1: The frequency of clinicopathological features in colorectal cancer samples

\begin{tabular}{lccc}
\hline Variables & Factor & N & \% \\
\hline \multirow{2}{*}{ Age } & $<=50$ & 12 & 20 \\
\cline { 2 - 4 } & $>50$ & 48 & 80 \\
\hline \multirow{2}{*}{ Sex } & Male & 39 & 65 \\
\hline \multirow{2}{*}{ Type of tumor } & Female & 21 & 35 \\
\hline \multirow{2}{*}{ Location of tumor } & Mucinous & 10 & 16.7 \\
\cline { 2 - 4 } & Non-mucinous & 50 & 83.3 \\
\hline \multirow{3}{*}{ Stage } & Colon & 41 & 68.3 \\
\cline { 2 - 4 } & Rectosigmoid & 19 & 31.7 \\
\hline \multirow{3}{*}{ Grade } & I & 20 & 40 \\
\cline { 2 - 4 } & II & 23 & 46 \\
\cline { 2 - 4 } & III & 7 & 14 \\
\hline \multirow{2}{*}{ Lymph node metastasis } & I & 8 & 13.3 \\
\cline { 2 - 4 } & II & 42 & 70 \\
\hline
\end{tabular}

through the downregulation of tumor suppressor genes. ${ }^{6}$ It is reported that tumor suppressor genes are progressively being inhibited in CRC. ${ }^{7}$ Screening of tumor suppressor genes in metastatic $\mathrm{CRC}$ has revealed that methyl binding protein (MBD) 1 gene, compared with other genes that were screened for methylation levels, had the most downregulated mRNA expression and upregulated methylation levels in advanced CRC. This upregulation in methylation level continued along with the progression of CRC. ${ }^{8}$ MBP family is characterized by its interaction with methylated DNA. ${ }^{9}$ Since the identification of the first MBP in 1989, MeCP2\&1, their numbers have increased and 15 MBP have already been known in human body, which are splitted into three branches. ${ }^{10}$ The genes MBD1 and MBD2 encode methyl-CpG binding proteins that suppress transcription from methylated promoters. ${ }^{11}$ Nevertheless, the oncogenic role of MBD1 is not typical for all cancers. In human pancreatic carcinomas with lymph node metastasis, for instance, elevated expression of MBD1 has been reported. ${ }^{12,13}$ Furthermore, knockdown of MBD1 prevented the invasion and cell proliferation of pancreatic cancer cells. ${ }^{14}$ Whereas, in acute promyelocytic leukemia, ${ }^{15}$ prostate cell line, and colon cancer cell lines, depressed expression of MBD1 has been reported. ${ }^{8}$ It also has been suggested that MBD1 may act as tumor suppressor in advanced form of $\mathrm{CRC}$ and have impacts on the development of metastasis by regulating other tumor suppressor genes. ${ }^{7}$ In this study we aimed to assess the immunohistochemical expression of MBD1 in CRC, and evaluate how this expression is correlated to clinicopathological features of sex, age, location of tumor, stage, and grade to study the possibility of considering MBD1 as a marker of prognosis in CRC.

\section{MATERIALS AND METHODS}

\section{Case selection and tissue samples}

This research project was approved by the Ethics Committee of Isfahan University of Medical Sciences under the number \# 397382. A total of 60 samples of CRC from patients who underwent surgical gastroenterology operations (either in elective operations or emergency operations) at Alzahra Hospital (Isfahan, Iran), and 60 samples of normal adjacent tissue (more than $10 \mathrm{~cm}$ far from the margin of tumors) were analyzed in a retrospective longitudinal clinical study. They did not have any signs of degenerative chronic diseases. Cases with non-resected tumors and previous use of anti-neoplastic therapy were excluded.

Out of the 60 adenocarcinoma samples, 50 were mucinous and 10 were non-mucinous. 39 cases were from men (65\%) and 21 were from women (35\%). 53 had yielded through elective operations (88\%) and seven through emergency operations (12\%). The staging of the tumors was done using the Duke classification. The frequency of clinicopathological features is described in table 1.

\section{Immunohistochemistry}

All samples were subjected to immunohistochemical analysis of MBD1 for one tumor-rich section per case and one adjacent tumor-free section as a normal control for that case. Formalin-fixed and paraffin-embedded tissue specimens were used for immunohistochemistry (IHC). ICH was done based on the previously described protocol. ${ }^{2}$ Briefly, sections with $5 \mu \mathrm{m}$ thickness from paraffinembedded blocks were deparaffinized in xylene and rehydrated using a graded series of ethanol. In the next step, antigen retrieval was performed on the sections by pretreating with $0.01 \mathrm{~mol} / \mathrm{L}$ citrate buffered saline $(\mathrm{pH}$ 6.0) and autoclaving at $121^{\circ} \mathrm{C}$ for $15 \mathrm{~min}$. Endogenous peroxidase activity was blocked by incubation with $3 \%$ $\mathrm{H}_{2} \mathrm{O}_{2}$ for $30 \mathrm{~min}$ at room temperature. To block non- 


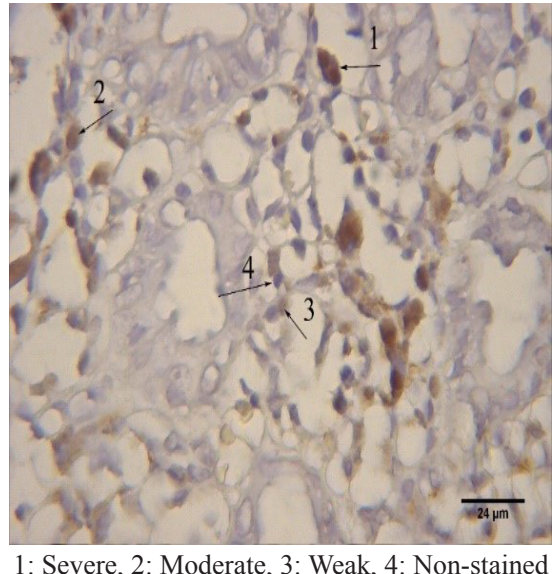

Fig.1: Intensity of MBD1 stained cells

specific binding of the immunological reagents, the sections were then incubated with $10 \%$ normal goat serum for $1 \mathrm{~h}$. After incubation with MBD1 antibody (\#ab238760, Abcam) at $4^{\circ} \mathrm{C}$ overnight, the peroxidase activity was developed with 3, 3'-diaminobenzidine tetrahydrochloride (DAB) in sterile $\mathrm{H}_{2} \mathrm{O}_{2}$ solution for 5 minutes. To counterstain nuclei, Mayer's hematoxylin staining was performed. Two blinded observers (M.A and Z.A) examined the immunostained sections independently.

\section{Cell counts and scoring system}

Stained tumor and normal adjacent sections were observed by a light microscope and at least 500 in 10 different fields were picturized by Motic Image Advanced Plus3. Cell count was done using the ImageJ software. A four-point scale was considered to evaluate the staining intensity of the sections as follows: $0=$ negative, 1 = weak, 2 =i ntermediate, and $3=$ strong (figure 1 ). The staining intensities were also verified by an independent observer. Total intensity per section was calculated by the following equation: $\mathrm{H}$-score $=\Sigma(1+\mathrm{i})$ pi.

\section{Statistical analysis}

Statistical analysis was performed by SPSS software version 25. Paired t test was used to compare protein expression between tumor and normal adjacent samples. Independent $\mathrm{t}$ test, Pearson correlation coefficient, and Spearman's rank correlation coefficient were used to evaluate the correlation between MBD1 expression and features of sex, age, location, type, grade, and stage of the tumor. Data were represented as mean $\pm \mathrm{SD}$ and $p$ values less than 0.05 were considered as statistically significant.

\section{RESULTS}

\section{General observation of samples}

Nuclear pattern with various staining intensities was observed as positive MBD1 staining in normal compared with negative unstained nuclei (figure 2).

A significant difference in MBD1 protein expression was noted in the tumor samples in comparison with the normal control samples (figure 3, table 2).

Correlation of MBD1 protein expression and clinicopathological features

Statistical analysis of the obtained data determined that there was a significant difference in MBD1 protein expression in cancerous samples in comparison with normal adjacent samples $(p<0.001)$. Of the 60 cases that were randomly selected, 39 cases were from male and 21 cases were female patients. No significant correlation was found between the expression of MBD1 protein and sex $(p=0.92)$. We also did not observe any significant correlation between MBD1 protein expression and age $(p=0.54)$. Regarding the type, location, stage, and grades of tumors, there were not any significant differences between cancerous and normal samples ( $p=0.82,0.75$, 0.87 and 0.80 , respectively). However, a significant reduction in MBD1 protein expression was seen in tumors that had metastasis to lymph nodes compared with those who did not have metastasis to lymph nodes $(p=0.04)$. The statistics are described in tables 3, 4, 5, 6, 7 and figure 4 .

\section{DISCUSSION}

Methyl-CpG binding proteins are characterized by their interaction with methylated DNA and are considered as interpreters of the DNA methylation signal. ${ }^{16}$ The MBD1 protein is the largest member of this family of binding proteins. The reports of MBD1 roles in cancer are not concordant. While some studies indicated the repressive action of MBD1 on some tumor suppressor genes and association of MBD1 with tumor metastasis, ${ }^{17}$ others suggested its tumor-suppressive roles in cancer. ${ }^{11}$ It has been suggested that MBD1 may also act as a tumor suppressor in CRC. Downregulation of MBD1 gene 

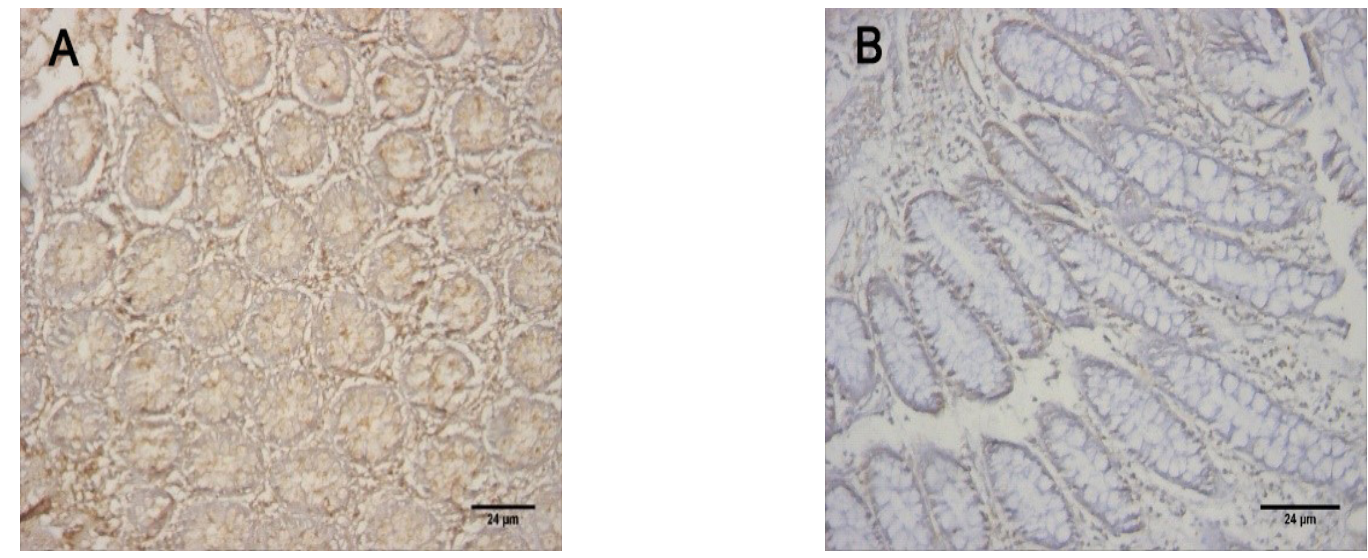

Fig.2: The MBD1 stained samples. Normal colon tissue (A) and cancerous tissue (B), (100X). The cancerous tissue showed significantly lower level of expression than normal tissue.

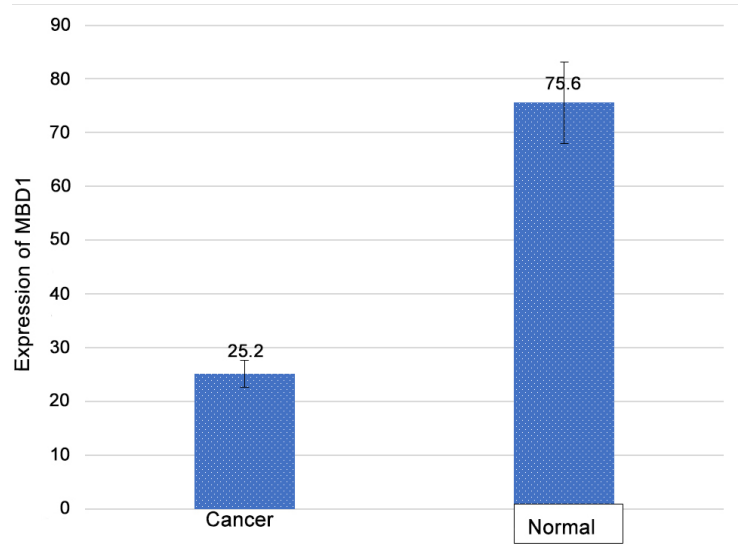

Fig.3: The mean expression of MBD1 protein in cancerous samples compared with normal samples

Table 2: The mean expression of MBD1 protein in cancerous samples compared with normal adjacent samples

\begin{tabular}{lccc}
\hline Variables & Mean & SD & p-value \\
\hline Cancer & 25.2 & 3.3 & \multirow{2}{*}{$<0.001$} \\
\cline { 1 - 3 } Normal & 75.6 & 5.7 & \\
\hline
\end{tabular}

Table 3: The expression of MBD1 protein expression correlation to gender

\begin{tabular}{lccccc}
\hline \multirow{2}{*}{ Variables } & \multicolumn{2}{c}{ Male } & \multicolumn{2}{c}{ Female } & \multirow{2}{*}{$\boldsymbol{p}$ - } \\
\cline { 2 - 5 } & SD & Mean & SD & Mean & \\
\hline Cancer & 24.9 & 3.9 & 25.7 & 6.1 & 0.92 \\
\hline Normal & 73.9 & 6.9 & 79.4 & 9.9 & 0.62 \\
\hline
\end{tabular}

Table 4: The Pearson correlation coefficient of MBD1 protein expression correlation to age

\begin{tabular}{lcc}
\hline \multirow{2}{*}{ Variables } & \multicolumn{2}{c}{ Age } \\
\cline { 2 - 3 } & $\mathbf{r}$ & $\boldsymbol{p}$-value \\
\hline Cancer & 0.082 & 0.54 \\
\hline Normal & -0.071 & 0.59 \\
\hline
\end{tabular}

35

30

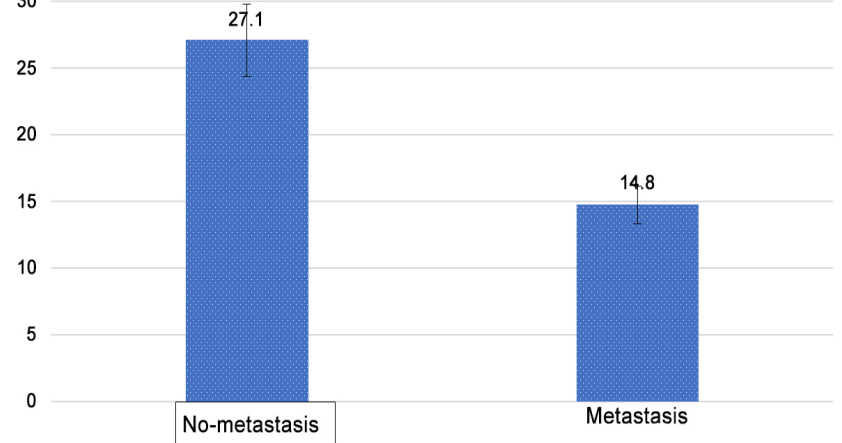

Fig.4: The mean expression of MBD1 protein in cancer samples compared to normal samples correlation to tumor type

Table 5: The mean expression of MBD1 protein in cancer samples correlation to tumor type

\begin{tabular}{lccc}
\hline Tumor Type & Mean & SD & $\boldsymbol{p}$-value \\
\cline { 1 - 3 } Mucinous & 23.5 & 9.9 & \multirow{2}{*}{0.82} \\
\cline { 1 - 3 } Non-mucinous & 25.6 & 3.5 & \\
\hline
\end{tabular}

Table 6: The mean expression of MBD1 protein correlation to tumor location

\begin{tabular}{lccc}
\hline Tumor Location & Mean & SD & $p$-value \\
\hline Colon & 24.5 & 4.1 & \multirow{2}{*}{0.75} \\
\cline { 1 - 3 } Rectosigmoid & 26.8 & 5.5 & \\
\hline
\end{tabular}

Table 7: The mean expression of MBD1 protein correlation to lymph node metastasis

\begin{tabular}{lccc}
\hline Lymph node metastasis & Mean & SD & p-value \\
\cline { 1 - 3 } Yes & 27.1 & 3.8 & \multirow{2}{*}{0.04} \\
\cline { 1 - 3 } No & 14.8 & 3.6 & \\
\hline
\end{tabular}


was seen along with the progression of metastatic CRC that was mediated by hypermethylation of this gene. ${ }^{8}$ In this present study, we investigated the immunohistochemical expression of MBD1, to evaluate its expression alteration in CRC and assess the correlation of its expression to clinicopathological features to study the possibility of considering MBD1 as a marker of prognosis in CRC.

The results of IHC of MBD1 showed that the expression of this protein was significantly lower in cancerous cases compared with the adjacent control samples. This finding is in agreement with the previous enrichment analysis study that suggested that MBD1gene had downregulated mRNA expression and continuously upregulated methylation levels in CRC. ${ }^{8}$ Regarding the correlation between MBD1 protein expression and clinicopathological features, no correlation was found between MBD1 expression and sex, age, type, and location of the tumors. We also did not find a significant difference in MBD1 protein expression between the stages of non-mucinous tumors, nor between the grades of mucinous tumors, while it has been suggested that MBD1 gene mRNA expression continues to downregulation with CRC progression. ${ }^{8}$ However, among our cancerous cases with metastasis to lymph nodes, MBD1 expression was significantly lower than those without metastasis to lymph nodes. This discrepancy may be due to the sample size used in this study and may be explained more confidently with larger sample size.

\section{CONCLUSION}

The results of this study suggest that MBD1 is involved in the pathogenesis of CRC. Based on the results of this study and considering the sample size, MBD1 protein is not a good predictor for CRC prognosis.

\section{ACKNOWLEDGMENT}

This study has been supported by grant No. 397382 from Research Deputy of Isfahan University of Medical Sciences.

\section{ETHICAL APPROVAL}

There is nothing to be declared.

\section{CONFLICT OF INTEREST}

The authors declare no conflict of interest related to this work.

\section{REFERENCES}

1. Ghavam-Nasiri M-R, Rezaei E, Ghafarzadegan K, SeilanianToosi M, Malekifard H. Expression of p53 in colorectal carcinoma: correlation with clinicopathologic features. Arch Iran Med 2007;10:38-42.

2. Akshatha C, Mysorekar V, Arundhathi S, Arul P, Raj A, Shetty S. Correlation of p53 overexpression with the clinicopathological prognostic factors in colorectal adenocarcinoma. Journal of clinical and diagnostic research: $J$ Clin Diagn Res 2016;10:EC05-EC08. doi: 10.7860/ JCDR/2016/22617.9056

3. Georgescu CV, Saftoiu A, Georgescu CC, Ciurea R, Ciurea T. Correlations of proliferation markers, p53 expression and histological findings in colorectal carcinoma. $J$ Gastrointestin Liver Dis 2007;16:133-9.

4. Hong SN. Genetic and epigenetic alterations of colorectal cancer. Intest Res 2018;16:327-37. doi:10.5217/ir.2018.16.3.327.

5. Coppedè F, Lopomo A, Spisni R, Migliore L. Genetic and epigenetic biomarkers for diagnosis, prognosis and treatment of colorectal cancer. World J Gastroenterol 2014;20:943-56. doi: 10.3748/wjg.v20.i4.943.

6. Llinas-Arias P, Esteller M. Epigenetic inactivation of tumour suppressor coding and non-coding genes in human cancer: an update. Open Biol 2017;7. pii: 170152. doi: 10.1098/rsob.170152.

7. Wang LH, Wu CF, Rajasekaran N, Shin YK. Loss of Tumor Suppressor Gene Function in Human Cancer: An Overview. Cell Physiol Biochem 2018;51:2647-2693. doi: 10.1159/000495956.

8. Qi L, Ding Y. Screening of Tumor Suppressor Genes in Metastatic Colorectal Cancer. Biomed Res Int 2017;2017:2769140. doi: 10.1155/2017/2769140.

9. Bland JM, Altman DG. Multiple significance tests: the Bonferroni method. BMJ 1995;310:170.

10. Hintikka J, Hintikka KJJ, Hintikka MB. The logic of epistemology and the epistemology of logic: selected essays: Springer Science \& Business Media; 1989.

11. Parry L, Clarke AR. The roles of the methyl-CpG binding proteins in cancer. Genes Cancer 2011;2:618-30. doi: 10.1177/1947601911418499.

12. Mahmood N, Rabbani SA. DNA Methylation Readers and Cancer: Mechanistic and Therapeutic Applications. Front Oncol 2019;9:489. doi: 10.3389/fonc.2019.00489.

13. Mahmood N, Rabbani SA. DNA methylation and breast cancer: mechanistic and therapeutic applications. Trends Cancer Res 2017;12:1-18.

14. Zhu H, Wang G, Qian J. Transcription factors as readers and effectors of DNA methylation. Nat Rev Genet 2016;17:551-65. doi: 10.1038/nrg.2016.83. 
15. Villa R, Morey L, Raker VA, Buschbeck M, Gutierrez A, De Santis F, et al. The methyl-CpG binding protein MBD1 is required for PML-RAR a function. Proc Natl Acad Sci U S A 2006;103:1400-5. doi:10.1073/pnas.0509343103.

16. Jørgensen HF, Bird A. MeCP2 and other methyl-CpG binding proteins. Ment Retard Dev Disabil Res Rev 2002;8:87-93.

17. Xu J, Zhu W, Xu W, Yao W, Zhang B, Xu Y, et al. Upregulation of MBD1 promotes pancreatic cancer cell epithelial-mesenchymal transition and invasion by epigenetic down-regulation of E-cadherin. Curr Mol Med 2013;13:387-400. 